\title{
THE AUTOMATION EQUIPMENT IN THE PALLETIZING WORKPLACE IN THE INTELLIGENT ASSEMBLY CELL
}

\author{
SEBENOVA, S[ilvia] \& VELISEK, K[arol]
}

\begin{abstract}
Intelligent assembly cell conception includes new solution kind of how to create structures of automated and flexible assembly system. Intelligent behavior of the system as the control system will repose on monitoring of important parameters of the system in the real time. The design of palletizing workplace with industrial robot IRB 120, specifically in the design of the automation equipment is based on the possibility of the application, use and functionality of the elements present in the workplace already has.

Keywords: assembly, intelligent assembly cell, automation, palletizing workplace, industrial robot IRB-120
\end{abstract}

\section{INTRODUCTION}

When analyzing the current state of palleting workplace is to be understood it as part of the palleting work of intelligent assembly cell, which is located in the laboratory UVSM MTF STU Trnava.

Intelligent assembly cell is one of the most common design of cellular manufacturing. It forms the basic element in the application of flexible automation for small and medium-sized parts. Assembly is carried out automatically in a limited time and unattended. The construction is based on the fundamental elements of automation and robotics. These are the building blocks that can be changed to some extent and thus create the corresponding device [1].

Intelligent assembly cell is a system consisting of: sub-assemblies (used for automatic mounting of basic elements in sets, or groups to higher assemblies, which provide for automatic exchange of positions and compensation instruments), handling subsystem (performed in addition to handling operations and activities associated with automatic exchange of assembled objects, activities, transportation in a buffer vstupnovýstupnom place cell handling devices, and waste) and control subsystem (its function is to control, coordination, synchronization and monitoring functions of cell production) [2].

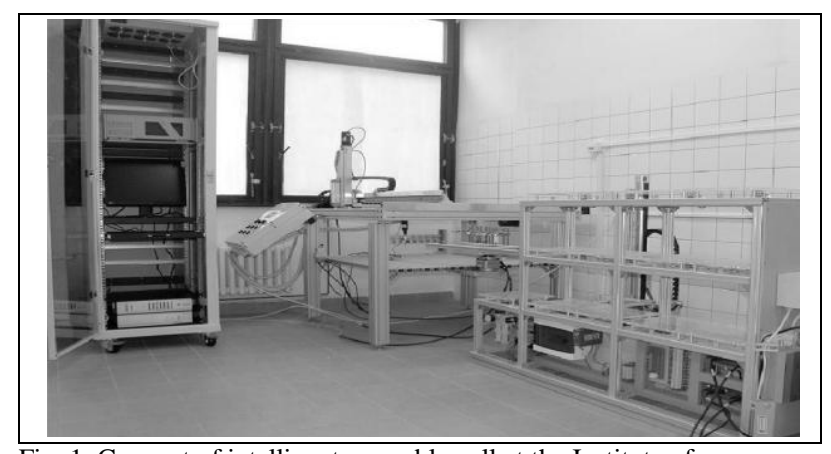

Fig. 1. Concept of intelligent assembly cell at the Institute of Production Systems and Applied Mechanics

\section{DESIGN OF INTELLIGENT ASSEMBLY CELL}

In Fig. 2 is a schematic design of intelligent assembly and /or disassembly cell, which consists of the following subsystems: storage system, rotary feeder, Cartesian robot with automatic exchange of grippers, the mobile robot Robotino and palleting workplace with industrial robot IRB 120 [3].

The sequence of operation and functions of various departments in intelligent assembly cell is as follows. Shelf storage system, which includes pallet stacker, serves as intermediate storage, the individual components and assembled products. Prefabricated components are placed on pallets with binary code. The pallets are placed in three rows and five columns, while the bottom row are six pallets.

Storage handler selects and delivers a variety of board from the bottom shelf of the rotary feed device or the input / output space for robot Robotino. After the arrival of pallet to the rotary table, rotary table places the pallet in a workspace of multi-functional Cartesian robot. That realizes the assembly or disassembly of springs or lids to different types of rollers. After the technological operations and follow-up, the pallet of returns back through the rotary table to the shelf stacker. The next operation is performed on the input / output location for the robot Robotino. This ensures conveying between the pallet stacker and pallet workplace with industrial robot IRB 120.

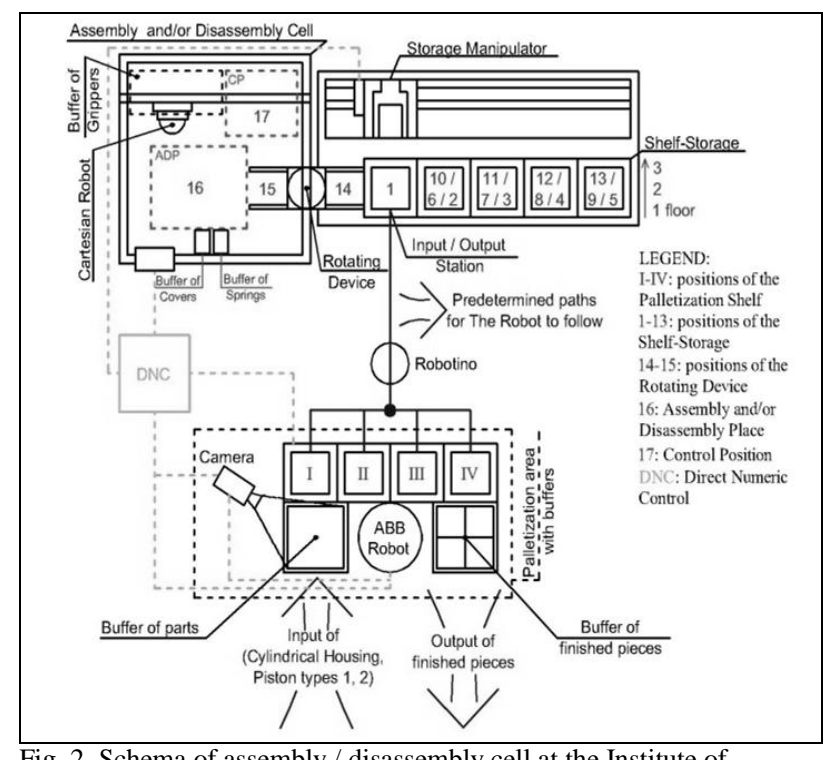

Fig. 2. Schema of assembly / disassembly cell at the Institute of Production Systems and Applied Mechanics [2] 
The control of intelligent assembly and / or dissasembly cell is integrated into the central controling unit of the DNC, which uses pneumatic, electro and electronic control elements.

\subsection{Industrial robot IRB 120}

Industrial robot with the type designation IRB 120 is the smallest robot from ABB. This is a 6 - axis industrial robot with a compact design and high precision and flexibility. Kinematics of the robot consists of six rotary kinematic pairs and in terms of the overall kinematics of the robot is an angular structure.

Due to its small size, low weight, high flexibility and accuracy of positioning is designed especially for handling applications with smaller objects, requiring high accuracy of positioning in a confined urban space. The robot at high speeds, is able to achieve during their activities, you can maintain a high positioning accuracy. The combination of these properties with the ability to operate in small or confined spaces, which are the parameters of the robot (compact base dimensions) is designed to work in small manufacturing cells. Accordingly, fit well with the concept of intelligent cells at the Institute of Production Systems and Applied Mechanisc as a core element pallet work.

Allows multiple mounting options from the current (on base) to strengthen angle or the ceiling. With its compact body can be placed robot, as close to the production process and thereby reduce production cycle times [4].

The IRB 120 is equipped with technology QuickMove, TrueMove and SoftMove, forming part of the software equipment. QuickMove technology uses complete dynamic model of the robot control system. Optimizes the speed and acceleration of the robot and it is possible to maximize the robot acceleration for movements using primarily one axis and reach the final position as soon as possible, implying that the time to reach the end point depends not only on the velocity. TrueMove ensures that there is no change in the trajectory of the arm when changing speed. TrueMove technology is used to optimize the accuracy of the trajectory. The technology allows SoftMove "release" the robot in a straight line, which serves to eliminate inaccuracies in positioning.

In terms of ecology, the robot is environmentally friendly because it has a low energy consumption for its operation and use of electricity. Furthermore, the control periphery of the robot contains connectors for compressed air and electrical signals conducted inside the robot body. Connectors for these lines are in two places the robot: the back of the robot base and the top of the flange. In Fig. 3 are details Integrated connectors for power (return) signals (1) and for compressed air (2).

They are used to control the gripper and robot peripherals. The standard software equipment of the robot is to support the programming modes on-line and off-line. For off-line programming software is used by companies RobotStudio ABB, which

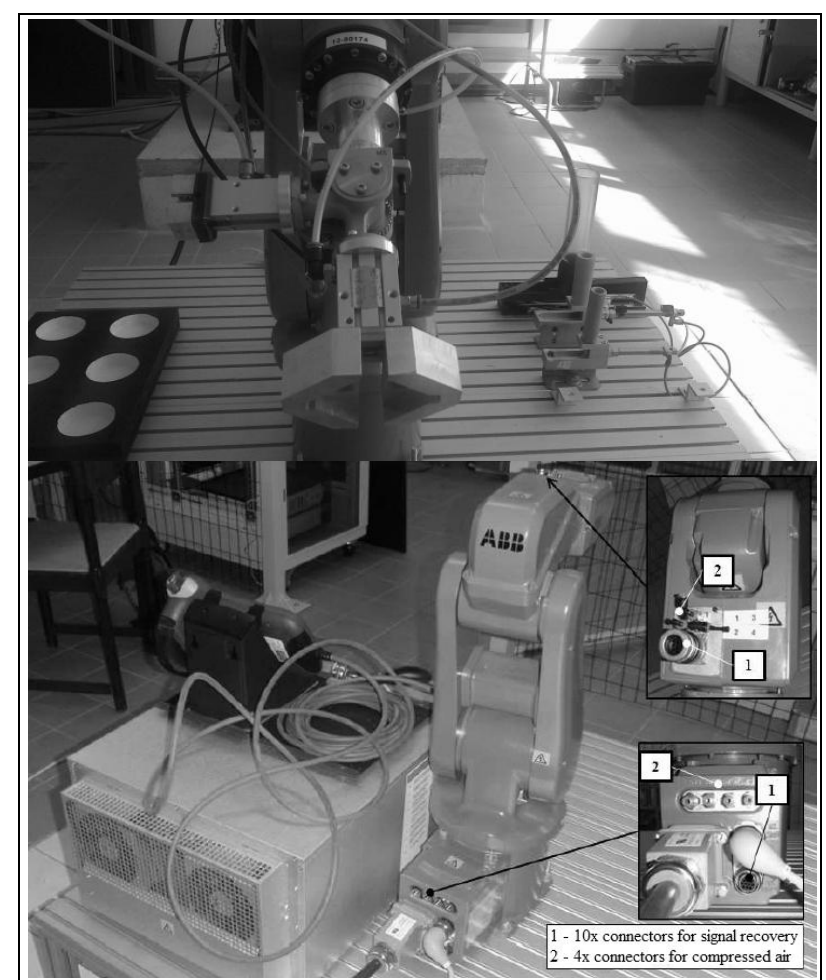

Fig.3 IRB 120 robot with a detailed view of integrated connectors

allows to transmit real data from your computer to compact IRC5 controller. The software can create (model) and simulate manufacturing processes (workplace) robot and work without interruptions or robot manufacturing process. It contains a database of robots, control systems and tools. Works with multiple robots, allows detection of conflict and dangerous conditions.

Programming on-line using a flexible programming unit FlexPendant IRC5 compact. This is a manual control panel used to carry out a wide range of tasks, functions and operations within the robot work. The main features include: run programs directly moving the 3D joystick manipulator and fine-tuning and modifying programs. FlexPendant is graphically transparent computer unit with a large color touch screen, which is easy to clean and highly resistant to external environmental factors (water, oil, etc.). It forms an integral part of the controller IRC5 compact. It consists of hardware and software parts. The software portion includes a transparent menu functions in several languages and programming language RAPID - the essential and major part of the robot software equipment.

The robot consists of a compact control system IRC5 compact, which includes all the standard features necessary to manage and control body motion robot (manipulator) while ensuring interaction with the control panel FlexPendant with the robot and peripherals. IRC5 control system is stored in a compact shielded chassis. IRC5 compact control system has two basic modules.

The management module contains all the control electronics, the main computer, network card, allowing connection of I / O using I / Q XS7 connector and flash memory. I / Q XS7 connector connects to 16 external inputs and 16 outputs outside the integrated network card 
DSQC 652. Jack XS7 works and processes signals with $24 \mathrm{~V}$. The control module runs the operation and all operations of all software needed to control the robot, which is basically a system RobotWare.

RobotWare is managing virtual robot controller, which in the off-line mode allows simulation program and on-line mode, RobotStudio software provides interaction with the physical control system. The most important part of the powerful and configurable software RobotWare is a programming language RAPID. RAPID is highly flexible and powerful tool for creating structured programs of different types of robotic workstations for example. for welding, handling of goods and others. In addition to the programming language RAPID system contains RobotWare QuickMove and technology, and TrueMove SoftMove.

The second module is the power (the drive) module containing all the power electronics is a source of energy for individual robot motors. The power module can handle up to nine power units and secure grasping in six axes. Each robot has its own power module that can be centrally managed through a control module with built MultiMove. Benefits IRC5 compact control system: the relatively low weight, dimensions, and the REASON easy handling and space saving, easy commissioning and attached external (peripheral) devices through the I / Q XS7 and plug the adapter directly DSQC 652 built-in control system [4].

\section{THE AUTOMATION ELEMENTS IN THE PALLETIZING WORKPLACE WITH INDUSTRIAL ROBOT IRB-120}

\subsection{The analysis of palletized/depalletized product}

In the workplace is carried out palletizing 5 types of components and finished depalletization (assembled) products. Parts from each other in certain differ characteristics (size, shape and material), they to be analyzed to identify the type components. Handling of components (Palletizing / depalletization) is performed by grasping the jaw effector for external cylindrical surface components (roller or pistil), so the basic dimensions of parts are outside diameter and height components. Parts must be in handling correctly oriented and positioned [5]. On individual pallets is necessary to store components to front side (without inner hole). The type of roller determine the based on information from the material (a combination of sensors), whereas all the rollers are in one container and have the same outer diameter. When palletization is required to meet the requirement of installation, and then palletize roller corresponding piston.

\subsection{The connecting plate}

To the mounting of the mechanical part of robot on the work desk is needed the connecting plate (Fig.4 ). It is used to secure and modular attachment that can not be achieved by direct anchoring (by bolting a $\mathrm{T}$ groove of working table). The connecting plate is made of structural steel, must be sufficiently strong and rigid to withstand the stresses and vibrations generated by moving the robot. The connecting plate has dimensions of $300 \times 300 \mathrm{~mm}$ and thickness $10 \mathrm{~mm}$. With four oval grooves with R4 and length $40 \mathrm{~mm}$ screw is not limited to position the robot in a direction perpendicular to the slot $\mathrm{T}$, the spacing between the grooves. The mechanical part of the robot is bolted to the connecting plate with four screws M12.

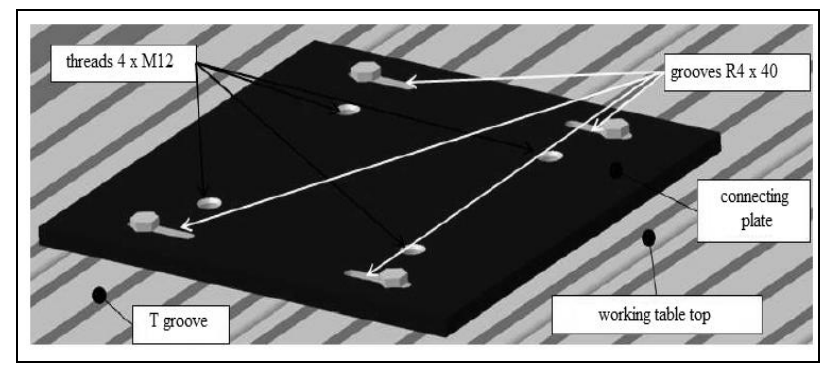

Fig.4. 3D model of the connecting plate

\subsection{Effector of a robot}

Output head of robot works with two types of components (cylinders or pistons), therefore has two grippers (gripper of rollers and the pistons). Both the gripper is located by two small flanges on the pointing devices. Pointing device has a range of rotation of present angle $\left(90^{\circ}\right)$, which is defined at both ends by mechanical stops, which is defined by its two positions. Pointing device is operated through a gear pneumaticly $5 / 2$ monostable valve. Both grippers are 2 spot with prismatic jaws. Pointing device is connected to the robot arm by two-flange. On the body of the third axis of the robot are connectors for compressed air and electrical signals, therefore be used for controlling the grippers (open / close) 5/2 monostable valves. On the connectors for the electrical signals are brought sensor signals of the end positions grippers and sensors to identify the type of roller, which are directly in the grippers of the rollers.

\subsection{Buffers}

Based on dimensional analysis of palletised components and of the proposed work cycles are needed at least 3 buffers and a maximum of 5. The stack for rollers is mounted to the work table directly using two Tnuts and bolts in the T-slots table. Buffers for the pistons are in the appropriate holders and they are fixed to work table by two nuts in T slots. All buffers are gravity with pneumatic feeders. Each pneumatic feeder (double acting linear pneumotor) is actuated $5 / 2$ monostable valve which is controlled electromagnetically (by coil). Final position of feeders are controlled by magnetic sensors. Speed Extension / retraction plunger feeders can be set using the throttle valve. [7]

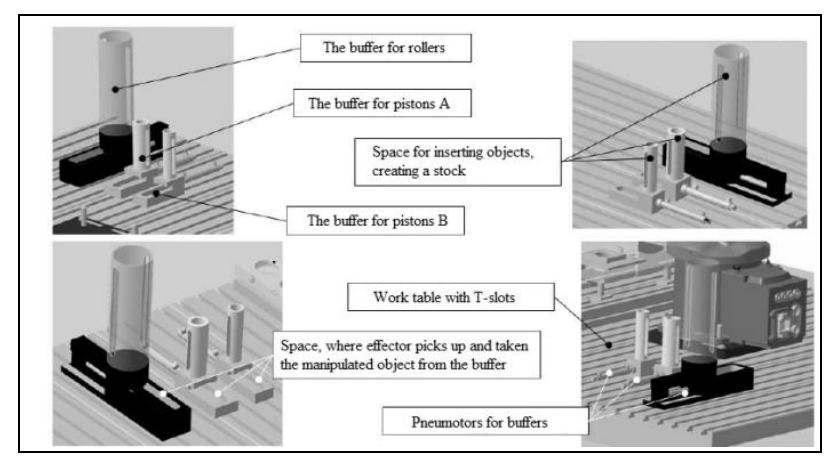

Fig.5 Characterization of buffers 


\subsection{Identification of the type of roller}

For the identification is necessary to determine the type of roller, respectively to meet the requirements for a particular type of palletized roller, when the tray randomly all three types of rollers.

Identification can be solved in two ways. The first solution is to identify the product by type of roller, what is created by identifying place directly on table. The sensors are screwed in two profiles L (induction - M12, optical - M18) so that they are rotated to each angle of 30 - (the elimination of mutual influence, smooth and quick access of affector to identification place). The whole preparation is fixed in $\mathrm{T}$ groove working table with a screw and T-matrix.

The second solution is to identify the type of roller directly in effectors. Both sensors with $\emptyset 4 \mathrm{~mm}$ are directly integrated into the jaws of the gripper for rollers. This results creating of a identification place directly in the jaws of the gripper for rollers.

\subsection{Pallets and storage space}

To palletizing and depalletization of components are on work desk these elements: the systems palette,the palette for finished products and storage space, which are designed based on the variants of operating cycles and requirements.

System palettes have a funkcion semitransport stackers. On the table top, on account of its size of range, 4 system paletts. Pallets are placed in front of the robot in its workspace in a row adjacent to the two profiles with base dimensions $40 \times 40 \mathrm{~mm}$ and a length of $1075 \mathrm{~mm}$. Profile bars are made of aluminum alloy and the like have a desk job board in the direction of the slot length $\mathrm{T}$, with which they are bolted to the counter table. Each palette is established on profiles and positioned with 4 pins and holes in the four L preparations. L preparations are associated with profiles with screws and $\mathrm{T}$ nuts in $\mathrm{T}$ slots. Pallets are made of plexiglass with dimensions: $245 \times 210 \times 10 \mathrm{~mm}$ and four aluminum pins with a tip height of $40 \mathrm{~mm}$. On the palette is attached the preparation to positioning roller and pistil of plexiglass with an internal diameter of $41 \mathrm{~mm} \varnothing$ (for rollers) and $\emptyset$ $20 \mathrm{~mm}$ (for pistons). [8]

In the each palette (at the palletizing) is a mechanical sensor (microswitch), serving on information the robot about state, which palette is occupied or empty, respectively. where the robot palletize the object, or possibly need to wait until the basic position is one of the pallet is released.

The palette for finished products is located on the table top in the robot workspace. It serves only to depalletization finished (assembled) products. It has six palletising seats for 6 finished (assembled) products. It is shape of a prism with a base measuring $260 \mathrm{~mm} \times 180$ $\mathrm{mm}$ and a height of $70 \mathrm{~mm}$. Finished products are stored in a pallete of progressively from first to sixth position, and by every time you save will remember the position occupied by the counter (counter). After saving the sixth finished product waiting robot in the rest position, until the full pallet of finished products exchanged for empty. The palette is in two parts: fixed and removable parts. The fixed part is fixed with screws and $\mathrm{T}$ nuts on the work table. Removable portion carries the finished products. Both parts are connected together shaped connections (four cone shape $\emptyset 20 \mathrm{~mm}$ with a shaped holes), which allows a quick exchange of pallets and is suitable for the automatic exchange of pallets by dedicated manipulator. To eliminate inaccuracies in positioning depalletization is in a storage location for each pallet of finished product, bevel edges $2 \mathrm{~mm} \times 45^{\circ}$.

\section{CONCLUSION}

Intelligent assembly cell conception, created for part production, or small series production, is an assembly system with some intelligent degree, which manipulate with semi-products and parts, which are assembled in the system to the final product [6].

The aim of this paper is to design of automation palletizing elements in intelligent assembly cell. Based on the requirements and analysis of the state have been proposed various automation components for palletizing workplace.

\section{ACKNOWLEDGEMENTS}

This paper was created thanks to the national grant: VEGA 1/0206/09 - Intelligent assembly cell.

\section{REFERENCES}

[1] Kovač J., Svoboda M., Liška O.: Automated and flexible assembly. Košice: Vienala, 2000. 375 s. ISBN 80-7099-504-1

[2] Ružarovský, R.,Šebeňová, S. Design of the sensory system in the intelligent assembly cell. In: Annals of DAAAM and Proceedings of DAAAM Symposium. ISSN 1726-9679. Vol. 22, No. 1. Annals of DAAAM for 2011 \& Proceedings of the 22nd International DAAAM Symposium "Intelligent Manufacturing \& Automation: Power of Knowledge and Creativity", Vienna, Austria. - Vienna : DAAAM International Vienna, 2011. ISBN 978-3-901509-83-4, p. 0095-0096

[3] Košt’ál, P., Mudriková, A Material and data flow in flexible manufacturing cell. In: Materials Science and Technology [online]. Roc. 7,. c. 3 (2007) [cit. 2012-01-10]. ISSN 1335-9053

[4] *** (2012) http://www.abb.com (2012) The ABB Group, Accessed on: 2012-01-28

[5] Danišová, N. \& Velíšek, K. (2009), Intelligent assembly system, Annals of MTeM for 2009 \& Proceedings of the 9th International Conference Modern Technologies in Manufacturing, 8th - 10th October 2009, Cluj-Napoca, Romania, Cluj-Napoca, ISBN $973-$ 7937-07-04, p. 57-60, Technical University of Cluj-Napoca

[6] Velíšek, K., Pecháček, F., Koštál, P., Štefanek, M.: (2005). Assembly machines and devices, STU, ISBN 80-227-2187-5, Bratislava

[7] Danišová, Nina - Majerík, Jozef: Jaw types design at the intelligent manufacturing-assembly cell., In: Annals of Faculty of Engineering Hunedoara - Journal of Engineering. - ISSN 1584 2673. - Tom IX, Fasc. 3 (2011), s. 279-281

Danišová, Nina - Majerík, Jozef: Sensoric system for identification of jaws in the jaw buffer and intelligent fixture., In: Annals of Faculty of Engineering Hunedoara - Journal of Engineering. - ISSN 1584-2673. - Tom IX, Fasc. 3 (2011), s. 441442 